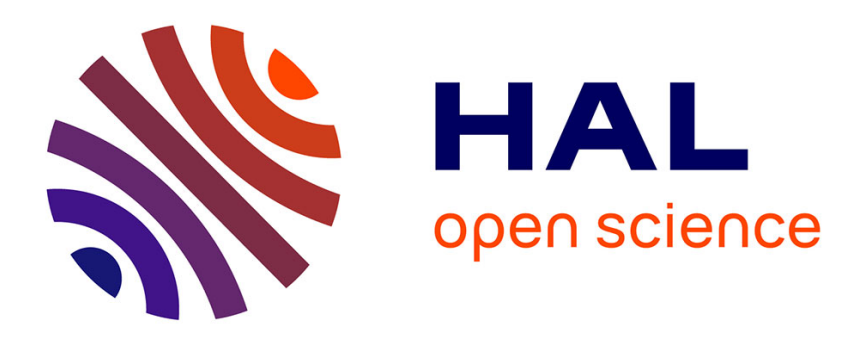

\title{
Images et perception de la présence divine en Grèce ancienne
}

\author{
Renée Koch Piettre
}

\section{To cite this version:}

Renée Koch Piettre. Images et perception de la présence divine en Grèce ancienne. Mélanges de l'Ecole française de Rome - Antiquité, 2001, 113 (1), pp.211-224. 10.3406/mefr.2001.10667 . halshs02433820

\section{HAL Id: halshs-02433820 \\ https://shs.hal.science/halshs-02433820}

Submitted on 9 Jan 2020

HAL is a multi-disciplinary open access archive for the deposit and dissemination of scientific research documents, whether they are published or not. The documents may come from teaching and research institutions in France or abroad, or from public or private research centers.
L'archive ouverte pluridisciplinaire HAL, est destinée au dépôt et à la diffusion de documents scientifiques de niveau recherche, publiés ou non, émanant des établissements d'enseignement et de recherche français ou étrangers, des laboratoires publics ou privés. 


\section{Images et perception de la présence divine en Grèce ancienne}

\section{Renée Piettre}

\section{Résumé}

Quels sont les rapports entre le texte et l'image dans les scènes d'épiphanies en Grèce ancienne ? La difficulté d'identifier une épiphanie divine dans l'image, notamment le risque de confusion entre l'humain et le dieu, a son correspondant dans les textes, non sans inflexions nombreuses : ces derniers compensent l'évanescence de l'apparition par son impact, et, selon le genre littéraire où s'inscrit l'épisode, selon les circonstances explicites de l'épiphanie (rêve ou veille, personnalité du bénéficiaire...), offrent des descriptions plus ou moins riches, non sans qu'il soit possible d'isoler un lexique spécifique et de cerner, en creux plus qu'en relief, des silhouettes reconnaissables. Mieux, le texte peut, comme l'image, présenter le dieu, le faire advenir dans et par le langage même, mimant ainsi l'efficacité du rite : cette fonction performative du rite transposée dans un art mimétique est analysée d'une part dans l'hymne de Callimaque Pour le bain de Pallas, d'autre part dans deux scènes de sacrifice sur des vases à figures rouges.

\section{Citer ce document / Cite this document :}

Piettre Renée. Images et perception de la présence divine en Grèce ancienne. In: Mélanges de l'École française de Rome. Antiquité, tome 113, n¹. 2001. Antiquité. pp. 211-224;

doi : https://doi.org/10.3406/mefr.2001.10667

https://www.persee.fr/doc/mefr_0223-5102_2001_num_113_1_10667

Fichier pdf généré le 16/09/2019 


\section{IMAGES ET PERCEPTION DE LA PRÉSENCE DIVINE EN GRÈCE ANCIENNE}

Introduire dans une recherche sur «Image et religion» l'épiphanie en Grèce ancienne ${ }^{1}$, c'est d'abord rappeler cette évidence, que représenter, c'est aussi rendre présent : dans l'épiphanie, justement, la divinité se rend authentiquement présente, du moins se la représente-t-on ainsi ${ }^{2}$. Ici, prend place tout un jeu entre le réel et l'illusion. Ce jeu est au fondement même de la croyance. C'est par lui et par la médiation du rite que se tissent les rapports unissant l'image cultuelle à la divinité destinataire du culte.

Le rapport entre l'épiphanie et l'image a fait l'objet d'un riche article de Burkhard Gladigow, Epiphanie, Statuette, Kultbild. Griechische Gottesvorstellung im Wechsel von Kontext und Medium ${ }^{3}$. Un autre article, de A. S. Versnell, What did ancient man see when he saw a god? Some reflections on greco-roman Epiphany ${ }^{4}$, pose de la manière la plus directe la question de l'expérience de l'épiphanie. Or, quand on juxtapose les deux auteurs, on constate qu'ils ne parlent nullement de la même chose. B. Gladigow s'interroge sur la médiation des images dans la représentation de la présence des dieux, à partir d'une documentation qui va de la période minœnne-mycénienne jusqu'à la Grèce archaïque et classique, alors que les exemples d'A.S. Versnell sont des documents écrits et nettement plus tardifs (essentiellement d'époque romaine). D'autre part, et surtout, B. Gladigow aborde l'épiphanie à partir du concept qu'en a formé l'histoire reli-

${ }^{1}$ Cf. R. Piettre, Le corps des dieux dans les épiphanies divines en Grèce ancienne (thèse EPHE, 5e section, dir. M. Detienne, 1996); Id., Les dieux crèvent les yeux : l'enargeia dans la représentation du divin, dans Ateliers : cahiers de la Maison de la recherche/Université Charles de Gaulle-Lille III, 21, 1999, p. 11-21.

${ }^{2} \mathrm{Cf}$. quelques pages suggestives de $\mathrm{D}$. Coppet, De l'action rituelle à l'image, représentations comparées, dans Philosophie et anthropologie, Paris, 1992, p. 115-130 (Espace international philosophie).

${ }^{3}$ Dans Visible religion, VII, 1990, p. 98-121, à compléter par, du même, Präsenz. der Bilder, Präsenz der Götter, dans Visible Religion, IV-V? (Approaches to iconology)?, 1985-1986, p. 114-133.

${ }^{4}$ Dans Dirk Van der Plas (éd.), Effigies dei, Leyde-New York, 1987, p. 42-55. 
gieuse, alors qu'A. S. Versnell se fonde sur le sens qu'il attribue au mot grec $\dot{\varepsilon} \pi \imath \varphi \alpha ́ v \varepsilon \imath \alpha$ (apparitions personnelles du dieu et/ou miracles qui lui sont attribués), apparu, selon notre documentation, dans un sens religieux qui couvre, par-delà l'apparition divine, toute sorte de miracles, en 278 av. J.-C., dans une inscription de Cos évoquant les prodiges ou les apparitions qui l'année précédente détournèrent les Galates du sanctuaire delphique $^{5}$.

\section{Épiphanie et image}

Le concept d'histoire religieuse que nous nommons épiphanie ${ }^{6}$, dis-

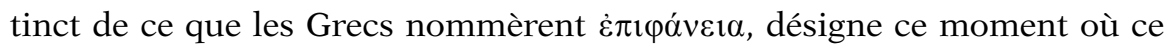
que perçoit le fidèle est intimement pour lui, et, si le contexte est rituel, de façon évidente pour la collectivité réunie, tout ou partie du dieu lui-même, le sûr indice de sa présence réelle et le corps où cette présence se donne à saisir. Dans l'épiphanie, le dieu est donc tenu pour réellement présent; cependant il ne s'agit jamais pour autant du dieu tel qu'en lui-même, mais de ce qu'il est censé laisser voir de lui en cette occasion-là, de la forme qui le représente et le présentifie en focalisant de vives émotions.

Une image cultuelle est le dieu présent, deus manifestus ac praesens, tantôt en des circonstances rituellement construites, au moyen des paroles, des gestes et des accessoires, tantôt grâce à l'irruption d'un miracle qui l'anime subitement; c'est si vrai que l'image n'est d'ailleurs pas indispensable. L'accessoire, ou un trône vide, la simple définition du lieu de la présence, pourvu qu'une mise en scène l'anime pour créer le moment épiphanique, peuvent remplacer l'image. Inversement, un prêtre ou un fidèle revêtus des attributs du dieu peuvent l'incarner avantageusement, sous sa forme animée, le temps du rite ${ }^{7}$. Dans l'iconographie mimétique, la représentation

${ }^{5}$ Syllogés, 398. Cf. à ce sujet G. Nachtergael, Les Galates en Grèce et les Sôtéria de Delphes. Recherches d'histoire et d'épigraphie hellénistiques, Bruxelles, 1977.

${ }^{6}$ Voir F. Pfister s. v. Epiphanie, dans RE, Supplement IV, 1924, col. 277-323; Е. Pax, ЕПІФАNЕIA. Ein religionsgeschichtlicher Beitrag zur biblischer Theologie, Munich, 1955; du même, s. v. Epiphanie, dans Reallexikon für Antike und Christentum, V, 1962, p. 832-909; D. Lührmann, Epiphaneia. Zur Bedeutungsgeschichte eines griechischen Wortes, dans G. Jeremias, H.-W. Kuhn et H. Stegemann (éd.), Tradition und Glaube. Das frühe Christentum in seiner Umwelt [Mélanges G. Kuhn], Göttingen, 1971, p. 185-199; D. Wachsmuth, Epiphanie, dans Der kleine Pauly, 5, 1975, p. 15981601; H. Cancik, s. v. Epiphanie/Advent, dans H. Cancik, B. Gladigow et M. Laubscher (éd.), Handbuch religionswissenschaftlicher Grundbegriffe, 2, Stuttgart-BerlinCologne, 1990, p. 290-296.

${ }^{7}$ On s'est volontiers imaginé sous cette forme certains rites extatiques de la Crète minoenne : cf. R. Hägg, Die göttliche Epiphanie im minoischen Ritual, dans 
d'une image cultuelle devra de toute façon utiliser certains traits ou signaux conventionnels si elle prétend figurer le moment «épiphanique». Par le rite, loin de toute illusion anthropomorphe, une divinité peut apparaître dans l'acte même qui la constitue : on fait surgir un dieu en habillant un poteau, une colonne ou un tronc (Dionysos jusqu'aux temps classiques ${ }^{8}$, ou encore les tropaia), ou quelque grossière poupée de bois; sur les images, suggère Gladigow, la présence de deux desservants de part et d'autre d'une divinité naissante ou épiphanique, notamment déployant étoffes ou vêtements (cf. Aphrodite sur le trône Ludovisi) est peut-être un indice de cette thématique. Le motif littéraire de la toilette du dieu (Aphrodite dans le $1^{\text {er }}$ et le $2^{\mathrm{e}}$ Hymne Homérique à Aphrodite) a pour répondant iconographique la représentation de la toilette rituelle d'une statue de culte; une telle représentation de la "présentification" rituelle du dieu se lit aussi dans le "geste épiphanique» des deux bras levés attesté dans l'iconographie ou la coroplastie créto-mycéniennes aussi bien que grecques, sans qu'on sache bien s'il s'agit de statues votives représentant le fidèle ou de statues cultuelles (ou plutôt de la représentation de l'une ou de l'autre), puisque le même geste peut appartenir à la divinité «épiphanique» ou à ses adorateurs saluant son épiphanie ${ }^{9}$.

Cependant un artiste, peintre ou sculpteur, peut naturellement aussi représenter sur le mode mimétique le dieu lui-même («en chair»), actif, présent et visible d'autres personnages représentés, hors de tout support

AM, CI, 1946; F. Matz, Göttererscheinungen und Kultbild im minoischen Kreta, Wiesbaden, 1958 (Abhandlungen der Geistes und Sozialwissenschaftlichen Klasse, 7). Pour la Grèce, voir par exemple le trône de Zeus au mont Ida (Porphyre, Vie de Pythagore, 17), un trône d'Apollon à Athènes (LSS 8, avec autres références à cet usage, p. 26). Cf. L. Weniger, Theophanien, altgriechische Götteradvente, dans Archiv für Religionswissenschaft, 22, 1923-1924, p. 16-57 surtout p. 37 sq. La mise en scène rituelle d'un trône vide est indirectement suggérée par une ruse d'Eumène de Cardia, Plutarque, Eumène 13, et Diodore de Sicile, XVIII, 60 sq.

${ }^{8}$ F. Frontisi-Ducroux, Le dieu-masque. Une figure du Dionysos d'Athènes, ParisRome, 1991 (Images à l'appui, 4).

${ }^{9}$ Ces rites supposent des images rudimentaires qui sont des supports occasionnels, parfois transportables et souvent pluriels (cf. les Daidala de Béotie, Pausanias IX, 3, 2-8), dont les groupes de divinités, Ilithyes, Mères etc., constituent peut-être, estime encore Gladigow (art. cit.), le souvenir à l'époque classique : à une époque toujours difficile à déterminer avec certitude, le passage de ces créations temporaires, à partir de supports neutres, à des images cultuelles fixes et souvent gigantesques, aurait instauré un tout autre rapport à la divinité désormais assignée à un lieu fixe, enfermée, tenue à distance, protégée et entretenue à grands frais, et dont l'accès est devenu éventuellement payant, et contrôlable. 
cultuel : quel est le rapport de cette illustration à l'image cultuelle vivifiée (ou non) par le rite? À quels indices pourra-t-on identifier la divinité d'une part (car l'anthropomorphisme divin la rend alors, en Grèce, difficilement discernable d'un personnage humain), sa qualité épiphanique de l'autre? Gladigow, pour répondre à cette question, se tourne vers les textes, que ce soit pour souligner la difficulté de la discrimination, ou pour suggérer des repères. Dans l'image, en cas de figures juxtaposées, on ne sait pas toujours très bien quel est l'homme et quel est le dieu. Cependant, une fois le dieu repéré (il a ses attributs, il surgit de lieux où on ne peut s'attendre à croiser un homme - la mer : Poséidon, Leucothéa, Athéna Aithyia; la montagne et les rochers : Pan; les profondeurs de la terre : Gê, Koré...-) les doutes sont rarement permis dans l'identification d'une épiphanie : la simple frontalité du dieu «en chair» (éventuellement flanqué d'animaux héraldiques ou d'acolytes), par rapport au spectateur de l'image, est tenue pour un marqueur conventionnel de l'épiphanie. La communication du dieu, par le regard et le geste, avec un spectateur situé dans ou hors de l'image signalent également l'épiphanie; dans ce cas, on rencontre éventuellement chez le spectateur l'expression de l'étonnement, de la stupeur, de l'éblouissement (bouche bée, regard détourné, main en visière... ${ }^{10}$ ). Associées à un tel signal, les ailes sont parfois un bon indice du dieu qui apparaît puis s'en va aussi vite qu'il est venu, dans l'image : elles sont généralement absentes des textes (sauf notamment pour des divinités allégoriques ${ }^{11}$ ou de fonction messagère).

Chaque medium a ses conventions propres. La taille et la beauté, l'éclat, le parfum sont associés au dieu dans les épiphanies littéraires, mais absents (parfum) ou non discriminatoires dans l'iconographie. Mais le medium capable de dire la difficulté de distinguer un dieu «épiphanique» d'un homme, de jouer de ce problème, c'est la parole et non l'image. Et si l'on veut comprendre comment les Grecs concevaient et percevaient l'apparition et la présence des dieux, les ressources des textes permettent d'éclairer les choix iconographiques, qui ne sont pas un décalque des choix linguistiques, mais relèvent plutôt des mêmes impératifs culturels.

${ }^{10}$ Pour ce dernier trait cf. I. Jucker, Der Gestus des Aposkopein. Ein Beitrag zur Gebärdensprache in der antiken Kunst, Zürich, 1956. Pour l'étonnement devant une assistance providentielle, on trouvera des exemples dans D. Wachsmuth, ПОМПI-

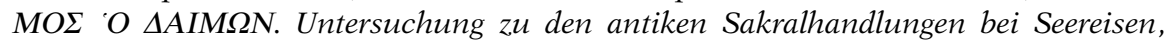
Berlin, 1967.

${ }^{11}$ Une Niké, un petit Hypnos recroquevillé ou un Eros, etc., divinités ou démons ailés, dans une image, ne sont pas à interpréter comme des épiphanies, mais servent de marqueur redondant pour l'identification de la scène représentée. 
L'épiphanie dans les textes

La possibilité de l'indécision entre l'identification d'un dieu ou celle d'un homme est particulièrement sensible dans les épisodes littéraires, voire historiques, de méconnaissance : scènes épiques ou tragiques où devant un être humain «beau et grand», un héros se demande s'il n'a pas affaire à un dieu (Ulysse et Nausicaa, Diomède et Glaucos ${ }^{12}$ ), ou inversement, est trompé devant un dieu déguisé (hôte qui vient éprouver la piété des hommes ${ }^{13}$, Dionysos face à Penthée ou aux pirates... $\left.{ }^{14}\right)$; mises en scène trompeuses qui font croire à l'arrivée d'un dieu (Pisistrate et Athéna, Hdt I, 60, 4; Messéniens et Dioscures, Paus. IV, 27, 2); métamorphoses déceptives, qui font que le plus habitué à leur fréquentation a toujours du mal à reconnaître les dieux (Ulysse et Athéna, Od. XIII, 312), lesquels du reste ne se laissent pas voir de tous (Od. XVI, 159-169 : Ulysse et les chiens voient Athéna, mais Télémaque ne la voit pas).

Déception : il n'y a rien à voir, ou si peu

Les textes insistent sur le caractère déceptif de la rencontre épiphanique : ou bien elle est insoutenable (fracas, éclat... «On ne peut regarder le soleil en face», rappelle Xénophon, Mémorables IV, 3, 12-14), ou bien elle est interdite, à tous ou de façon sélective (les bons seulement : Callimaque, Hymne à Apollon, v. 9), ou encore dangereuse (Thamyris, Tirésias, Actéon, Sémélé...). Jusque dans ses plus grandioses manifestations, on n'est jamais sûr de voir la divinité telle qu'en elle-même ${ }^{15}$ : elle prend tant de formes qu'on ne la reconnaît dans aucune. Ou bien ce n'est qu'à son départ qu'on la reconnaît, à la rapidité même de sa disparition, de son envol. Quant à cette apparence même, les textes en disent généralement fort peu : dans l'épopée, les dieux choisissent un corps, empruntent une identité ${ }^{16}$; rarement, ils laissent percer un peu de leur brillance et de leur éclat (Aphrodite au chant III de l'Iliade, v. 396-397) ou bien l'apparence choisie se rapproche à un moment donné, par le sexe ou l'allure, de celle qu'on peut attendre de

${ }^{12}$ Odyssée VI, 149-152; Iliade VI, 128-141.

${ }^{13}$ Exemples commodément réunis dans D. Flückiger-Guggenheim, Göttliche Gäste. Der Einkehr von Göttern und Heroen in der griechischen Mythologie, BernFrankfurt-am-Main-New York, 1984.

${ }^{14}$ Euripide, Bacchantes; Hymne homérique à Dionysos.

${ }^{15}$ N. Loraux, Qu'est-ce qu'une déesse?, dans dir. G. Duby et M. Perrot, Histoire des femmes. I. L'Antiquité, Paris, 1991, p. 31-62; l'auteur se demande s'il ne s'agit pas simplement d'une variante théomorphe des métamorphoses divines.

${ }^{16}$ J.-P. Vernant, Corps obscur, corps éclatant, dans Corps des dieux. Le temps de la réflexion, 7, 1986, p. 19-45. 
leur vrai corps (Athéna dans l'Odyssée, XVI, 157-158). On n'est pas plus renseigné pour autant. Ou bien on entend sans voir, on respire une odeur émanée d'une source invisible. Bref, la parole paraît souvent esquiver la peinture de l'épiphanie.

Mais il s'agit paradoxalement d'une déception comblée, d'un contentement frustré. Les textes évoquent à maintes reprises une sorte de dilatation de l'âme dans l'instant épiphanique (dans Homère : $\gamma \eta \dot{\eta} \theta \eta \sigma \varepsilon$, ió $v \theta \eta$ ). Nestor est aussi ravi que troublé lorsqu'il s'avise, au moment de l'envol de la déesse, qu'il fut l'hôte d'Athéna (Od. III, 373 sqq.). Le sourire d'Aphrodite est pour Sappho une consolation délicieuse (Sappho, fr. 1). Hippolyte ne sent plus ses blessures dans la proximité d'Artémis. Au combat, la présence divine protectrice rassure et galvanise ${ }^{17}$. Sur mer, si rapidement qu'elle passe, elle est providence et salut ${ }^{18}$.

L'épiphanie s'inscrit dans une expérience humaine de la durée : l'attente, le surgissement, l'arrachement à soi ou l'incertitude, l'instant qui comble, et déjà le départ, un parfum, une douceur qui s'attarde. Une conscience de soi apaisée. Il faudra en chercher la traduction iconographique.

\section{Le langage référentiel}

Mais cela ne nous donne guère prise sur le dieu. La perception est ici, pour le chercheur aussi, déception (et elle doit l'être, selon ce que nous avons dit de l'écart nécessaire entre le dieu tel qu'en lui-même et ce qu'il laisse voir de lui-même). Pour parvenir à cerner quand même le dieu dans ces moments de vision et d'intuition, il faut ruser avec les textes, faire flèche de tout bois.

Ce travail requiert d'abord une prise en compte de l'appartenance des documents à tel genre particulier : un texte épique expose des détails que l'on ne peut attendre d'un texte dramatique (les spectateurs voient, ils n'ont pas besoin de description) ou d'une texte historique (réduction ou disparition du merveilleux). La fiction permet d'affirmer plus fortement les représentations culturelles et leur laisse libre cours. Les textes épigraphiques se plient à une codification assez rigide et à une nécessaire brièveté. La relation historique ou biographique subordonne ces représentations à la fidélité au témoignage ou à l'événement, et laisse apparaître l'incertitude, le

${ }^{17}$ Nombreux exemples dans l'Iliade. Voir aussi W. K. Pritchett, The Greek State at war. III. Religion, Berkeley, Univ. of California Press, 1979, chap. 2.

${ }^{18}$ Nombreux exemples dans D. Wachsmuth, ПОМПIMOГ O $\triangle A I M \Omega N$. cit. n. 10 . 
flou, la contradiction. Hérodote est très laconique sur les apparitions en

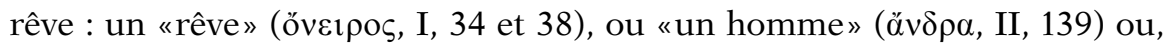

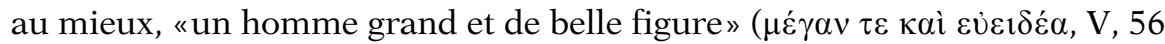

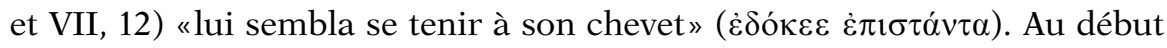
du Criton, Socrate dit avoir vue de même en rêve une «femme grande et belle». De quelle manière Apollon a-t-il secouru son sanctuaire delphique lors de l'attaque des bandes de Brennus? Est-il intervenu avec les «Vierges blanches» qui seraient, selon les uns, Artémis et Athéna (Justin 24, 8, 4-9, cf. Diod. 22, 9, 5), selon les autres, une personnification de la neige (ValèreMaxime I, 1, cf. Cic. De Divinatione I, 37, 81)? Ou bien n'a-t-on vu que les spectres de trois ou quatre héros (Paus. 10, 23, 2)? Ou bien tout simplement la panique des Gaulois a-t-elle été interprétée comme une manifestation de Pan, et leur mania, comme une manifestation d'Apollon (Paus. 10, 23, 7-8)? ${ }^{19}$

Tenons compte d'autre part des circonstances explicites de l'épiphanie. Il y a une grande différence entre une apparition óva (en rêve) et une apparition $0 \pi \alpha \rho$ dans la veille $)^{20}$. Les premières sont, dans les textes épigraphiques ou historiques, et aussi bien lyriques (Alcman fr. 47 P., Sappho fr. 134 L.-P.), celles du dieu, sans autre précision, sinon quelquefois l'indication de la statue cultuelle dont il arbore la figure (l'Asklépios de Pergame, Aelius Aristide, Discours sacrés, IV, 55; l'Athéna de Phidias, ibid. II, 41). Dans le même type de prose, les apparitions en veille sont celles d'un lièvreArtémis (Paus. III, 23, 6-7 et 22, 12), d'un serpent-Asklépios, de deux oies Isis et Sarapis (Aristide, Disc. sacrés III, 49), etc., à la rigueur celles d'un homme ou d'une femme de belle apparence (cf. Hdt VI, 61, apparition d'Hélène à Thérapné). Les stèles d'Épidaure (SIG $\left.{ }^{3} 1168-1169\right)$ accumulent les exemples dans les deux registres. A l'heure de midi cependant, au moment des siestes méridiennes, ou dans la nuit où les Muses visitent Hésiode ou Archiloque (inscription de Mnésiépès ${ }^{21}$ ), voire simplement dans quelque endroit retiré, un pâturage, une montagne, la pleine mer où surgissent les Dioscures secourables, les divinités affectent volontiers des formes plus surnaturelles, même devant un homme éveillé. Elles ne sont jamais aussi impressionnantes et redoutables que quand on les rencontre en plein combat. Décrivant l'épisode de Brennus à Delphes, Justin (24, 8, 4-9) écrit qu'au moment où le combat fait rage prêtres et devins «crient que le dieu est arrivé, qu'ils l'ont vu bondir dans le temple par le dôme entr'ouvert pendant que tous étaient prosternés pour implorer l'assistance du dieu, que

${ }^{19}$ Voir G. Nachtergael, Les Galates en Grèce, cit. n. 5.

${ }^{20}$ Cf. R. Piettre, Oneiros, le dieu-songe, dans Métis, 7, 1997, p. 115-140.

${ }^{21}$ Jacoby, FGrHist III b (Komm. Addenda), 415 sq. 
c'était un jeune homme d'une exceptionnelle beauté, plus qu'humaine, et que l'accompagnaient deux vierges en armes, qui avaient accouru des deux temples voisins de Diane et de Minerve; qu'ils n'avaient pas seulement de leurs yeux vu ces prodiges, qu'ils avaient entendu aussi le sifflement de l'arc et le bruit des armes». L'Athénien Polyzélos devint aveugle pour avoir vu lui apparaître en pleine bataille un phasma, une figure dont la barbe couvrait tout le bouclier (Souda, s.v. 'I $\pi \pi i \alpha \varsigma)$.

Une épiphanie est toujours fonction de la personnalité ou de la situation particulière du bénéficiaire (ou de la victime). Hippocrate le sait bien, qui diagnostique certains maux par l'identité des divinités agents de la possession pathogène, ou l'inverse (La maladie sacrée). Ulysse rencontre Athéna - quoi de plus logique? - comme Hector rencontre Apollon, et Énée Aphrodite. Comme le poète rencontre les Muses, et le malade Asclépios. Comme l'ennemi des Lacédémoniens trouve sur son chemin les Dioscures. La divinité rayonne autant à travers le corps de son protégé que par le sien propre. Y a-t-il d'ailleurs un seul bénéficiaire, ou plusieurs, ou tout un groupe, une armée?

Enfin, il convient de repérer le lexique spécifique et d'en analyser les

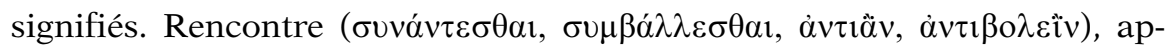
proche, proximité (le dieu se tient souvent tout près, cf. Odyssée : $\sigma \chi \varepsilon \delta$ óv,

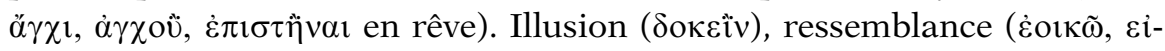

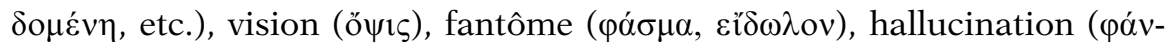
$\tau \alpha \sigma \mu \alpha)$, apparition claire ( $\varphi \alpha i ́ v \varepsilon \sigma \theta \alpha \iota \dot{\varepsilon} v \alpha \rho \gamma \varepsilon \imath \varsigma)$, présence et assistance $(\pi \alpha-$

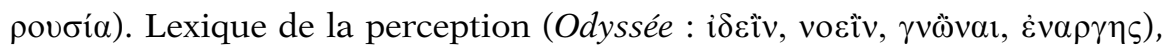

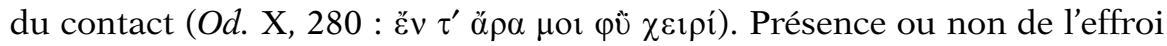

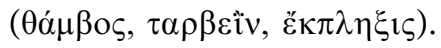

À l'intérieur de ce cadre, on peut alors à loisir repérer les plus petits détails descriptifs. Pour commencer, les registres sensoriels de la manifestation divine : quels sens, pour quels dieux? Puis les éléments comme la taille, la forme, l'espèce animale, le sexe, l'âge, les identités revêtues. Dans l'Hymne Homérique à Déméter, Déméter est d'abord une femme d'assez belle allure, mais au moment crucial sa tête touche le plafond. Dans Callimaque, elle touchera l'Olympe (Hymne à Déméter, 57-58). Dans l'Hymne Homérique à Apollon le dieu est d'abord dauphin monstrueux, puis astre en plein jour, puis robuste jeune homme dont les cheveux couvrent les larges épaules (400-450). Dans Apollonios de Rhodes, son arrivée provoque un séisme (Arg. II, 674-684). Une divinité féminine peut-elle revêtir des traits masculins (voir dans l'Odyssée Athéna en Mentor ou en berger)? Un dieu mâle peut-il se déguiser en femme? Si oui, lequel (laquelle)? On peut tenir enfin registre des parties du corps divin nommées dans le texte : quelles parties et pour quel dieu? Pourquoi les poèmes et les hymnes homériques 
évoquent-ils la peau d'Aphrodite, son $\chi \rho \omega ́ s$, ou son sourire, son regard, ses joues, sa gorge, son cou, ses genoux... mais, à part les yeux, rien de tout cela chez Athéna, seulement son vêtement ou son armement, et sa "lourde main»? Pourquoi les cheveux d'Apollon, ses épaules, rarement son regard?... Tenons compte aussi des cas où la divinité n'est pas nommée ni identifiée.

À faire ce travail on est conduit à se rendre à l'évidence que, comme Walter F. Otto le sentait bien ${ }^{22}$, ces dieux, même s'ils ne sont pas des «personnes", même s'ils se déclinent au pluriel et si beaucoup ne nous sont connus que par des noms, possèdent chacun dans l'imaginaire des Grecs une spécificité forte, qui renvoie chaque fois à une forme d'expérience, et qu'ils sont assez clairement silhouettés dans les représentations, même si des doutes sur leur identité se font jour, selon les textes, au moment de la rencontre. Au-delà de la juxtaposition des traits, ce qui permet de reconnaître l'individualité et l'unité de chacun relève d'ailleurs d'une perception plus floue et unifiante, d'une synesthésie fondée sur une connivence culturelle, dont la prise en compte est extrêmement délicate. Pour l'Iliade nous avons tâché ${ }^{23}$, par exemple, de saisir chaque dieu dans sa démarche et sa façon de s'inscrire dans l'espace, grâce à l'examen des verbes de mouvement utilisés, des parcours indiqués, des modes et des vitesses de locomotion, des attitudes, de la distance aux hommes. Cette espèce d'approche gestaltiste s'effectue aussi en creux, par une exploration de l'enveloppe aérienne du corps divin, du lieu, du cadre, de l'assistance, qui servent en quelque sorte de caisse de résonance à la venue et à l'action du dieu. Les réactions de la nature et de l'assistance animale, humaine, voire divine, permettent de mesurer les ondes émises et l'impact de l'épiphanie. Aphrodite arrivant sur l'Ida voit tous les grands fauves se couler vers elle et la flatter (Hymne Homérique à Aphrodite, 69-72), le gazon groît sous ses pieds (Hésiode, Théogonie, 194 sq.), Délos se couvre d'or sous les pas d'Apollon (Hymne Homérique à Apollon, 135-136). Devant la Déméter de Callimaque «demi-morts à sa vue, les gens d'Erysichthon s'enfuirent en hâte, laissant aux troncs les cognées» (v. 59-60).

C'est à travers tout cet ensemble que nous pouvons espérer, de façon moins directe qu'indirecte, appréhender quand même le corps et l'image des dieux, ne serait-ce qu'à travers les traces en creux de leur présence. C'est une thématique épiphanique importante, précisément, que celle des traces laissées par la divinité, preuves et monuments de son passage (cf.

${ }^{22}$ Walter F. Otto, Les dieux de la Grèce, trad. fr., Paris, 1981; Id., L'esprit de la religion grecque ancienne. Theophania, trad. fr., Paris, 1995.

${ }^{23}$ Dans notre thèse (voir note 1 ), I, A, 4 . 
Iliade 13, 44 sqq.). L'image peut-elle faire saisir cette complexité des épiphanies textuelles? Peut-elle faire voir sans montrer?

\section{Fonction symbolique et langage performatif}

Nous aurions tort en effet d'accuser le langage d'opacité. Puisqu'enfin ce sont ces mots mêmes, non moins que les rites, qui créent l'épiphanie. Eux, et le cadre parfois assez rigide que les conventions d'un genre, ou leur recréation, offrent à cette construction.

Dans l'épopée, les dieux apparaissent en contrepoint subtil aux actions des hommes, et ce sont souvent les mêmes «scènes-types» qui sont utilisées pour les hommes et pour les dieux qui se mêlent à eux (scènes d'ambassade, Botschaft; scènes de déplacement en char, Wagenfahrt; scènes de $\mu \varepsilon \rho \mu \eta \rho i \zeta \varepsilon 1 v$, inquiète méditation : cf. Walter Arend, Die typische Szenen bei Homer, Berlin, 1975 [1933]), en sorte que leur présence et leur action ne paraissent nullement détoner, d'autant plus que, non contents de se déguiser en hommes, ils investissent souvent les hommes de la ressemblance avec les dieux, au point que les hommes aussi peuvent susciter la panique (Iliade XVIII, 203-231 : Achille).

Les hymnes qui appellent le dieu à venir parmi ses fidèles le font venir en effet par cette invocation même. Leur langage est performatif. Le préfixe $\dot{\varepsilon} \pi l$ - qui apparaît dans $\dot{\varepsilon} \pi \iota \varphi \alpha ́ v \varepsilon ı \alpha$ se retrouve dans $\dot{\varepsilon} \pi 1 \kappa \alpha \lambda \varepsilon \varepsilon i ̈ v$, caractéristique de l'hymne "clétique», «appeler à venir», «inviter» (comme on invite un hôte à table), et le verbe a la même efficacité magique que غ̇ $\pi \dot{\alpha} \delta \varepsilon ı v$, «in-

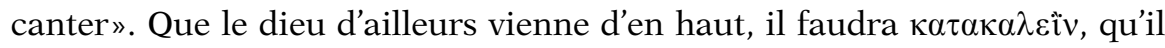

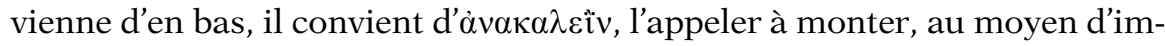

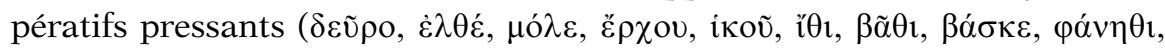
etc). Et de nombreux compléments d'origine pourront préciser le nom des sanctuaires qui font l'identité et le rayonnement du dieu, de même que les épiclèses, souvent tirées de toponymes, qui s'ajoutent aux épithètes de nature pour dessiner une figure et la susciter et la glorifier en même temps. Dans la tragédie, appelée par le chœur ou par un acteur, elle peut apparaître alors en effet, $\theta \varepsilon$ oì $\dot{\alpha} \pi \grave{~} \mu \eta \chi \alpha v \tilde{\eta} \varsigma$, ou bien se manifester de façon non moins évidente ou spectaculaire dans les souffrances des héros.

Parmi les hymnes «mimétiques» de Callimaque, les hymnes À Apollon et Pour le bain de Pallas recréent pour nous jusqu'à l'hallucination, grâce notamment à la complexité de l'énonciation, et le contexte rituel et le rythme de la fête et l'attente, l'appel, la venue du dieu, son entrée enthousiasmante ou sa présence : mais celle-ci est aussitôt distanciée ou rendue opaque par l'écran d'un arrière-plan mythique, qui permet à l'atemporel d'affleurer dans la durée humaine et de l'élargir démesurément en accueil- 
lant ce parfum d'immortalité, jusqu'à la prière apaisée qui salue la divinité. L'hymne Pour le bain de Pallas doit ainsi soutenir cette gageure, de faire assister, tout en la suscitant, à une épiphanie interdite, car le corps d'Athéna comme son visage et son regard ${ }^{24}$, qu'il s'agisse du Palladion d'Argos mené à son bain rituel, ou du «vrai» corps de la déesse, doit rester invisible ${ }^{25}$. Comment le présentifier néanmoins? En situant d'abord dans le temps mythique, et par la bouche de quelque participant(e) autorisé(e) de la fête qui s'adresse, en les pressant, aux jeunes $\lambda \omega \tau \rho \circ \chi$ óor argiennes chargées d'accompagner la déesse à son bain, quelques évocations précises des ablutions d'une Athéna sportive et combattante (qui songe d'abord à laver la sueur de ses chevaux, v. 9-12, comme le veut apparemment aussi le rite argien) et de son corps même, que sa seule pudeur protège encore : «Athéna... oignit son corps, en athlète expert, de l'essence toute pure que donne l'arbre qui est sien, Argiennes, et une rougeur montait à ses joues, comme on voit la rose matinale, comme on voit les grains du grenadier» (25-28). Puis vient le récit de l'aveuglement de Tirésias, qui «vit ce qu'on ne doit pas voir», le corps d'Athéna se baignant en des eaux courantes dans le silence de midi. L'apparition elle-même, dévoilement interdit aux regards de l'effrayant talisman, ou bien $\dot{\varepsilon} \pi \imath \delta \eta i_{\alpha} \alpha$ de la déesse elle-même, dans le temps rituel, se concentre dans les six derniers vers, ou plutôt dans le premier d'entre eux. En effet, le vers 147 saisit l'instant exact de l'apparition : 'E $\rho \chi \varepsilon \tau^{\prime}$ 'A $\theta \alpha v \alpha i \alpha$

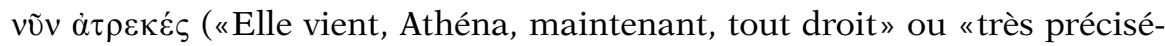
ment»). Nous sommes au moment où le char processionnel apparaît au bord de l'Inachos, mais ce moment est tout aussi bien senti comme une $\dot{\varepsilon} \pi \imath \eta \mu i ́ \alpha$, l'adventus de la déesse, sur un char aérien, parmi ses fidèles réunis pour son culte. Cet instant est pris dans un mouvement, un temps qui s'écoule (présent $\varepsilon \rho \chi \varepsilon \tau \alpha \imath$ ) et tout à la fois dans la fulguration du trait direct,

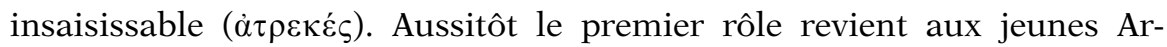

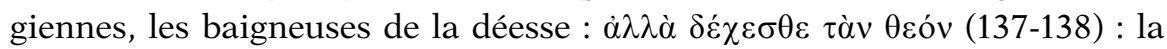
déesse étant là, il s'agit à la fois de l'accueillir comme déesse et de la récep-

${ }^{24}$ Cf. F. Frontisi-Ducroux, La face interdite, dans Id., Du masque au visage. Aspects de l'identité en Grèce ancienne, Paris, 1995, p. 63-75; L'oeil et le regard en Grèce ancienne, dans Iris (L'œil fertile), hors série, 1997.

${ }^{25}$ Voir A. W. Bulloch, The future of a Hellenistic illusion, dans Museum Helveticum, 41, 1884, p. 209-230; du même, Callimachus, the fifth hymn, Cambridge, 1985; Claude Calame, Quand dire c'est faire voir : l'évidence dans la rhétorique antique, dans Études de lettres, 4, 1991, p. 3-22; A. Henrichs, Gods in action: the poetics of divine performance in the hymns of Callimachus, dans M. A. Harder et alii (éd.), Callimachus, Groningen, 1993; N. Loraux, Ce que vit Tirésias, dans L'Écrit du Temps, 2, 1982, p. 109-116; K. J. Mc Kay, The poet at play. Kallimachos : the bath of Pallas, Leyde, 1962 (Mnemosyne, Suppl. 6). 
tionner comme image. Mais cette réception même est la condition de la présence divine, sa trace matérielle ou gestuelle. Or, comment reçoit-on la déesse? Ici le moyen et la manière semblent se résumer à l'accompagne-

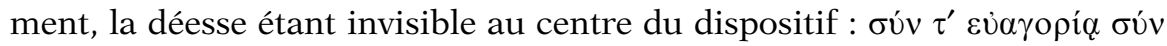

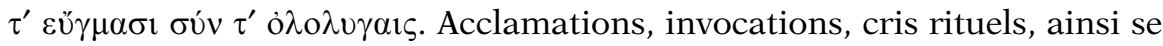
dit la présence et se trahit l'absence. Le texte, par l'anaphore, bat le pouls de l'émotion et le rythme du chant. Il mime l'instant paroxystique, et aussitôt, quand l'énonciateur, tourné vers la déesse, exprime à son tour, dans le salut qu'il lui adresse, l'évidence de sa présence, le salut tourne à l'adieu, au reflux, à la détente : Xaî̀ $\rho \varepsilon$ $\theta \dot{\alpha}$ (139), la déesse paraît saluée dans son appa-

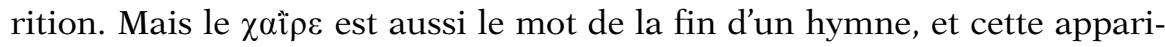

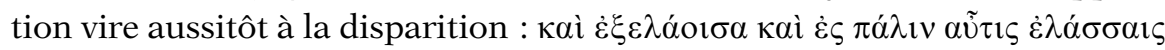
(140: «quand tu viens à nous et quand tu ramènes ton char»). Ainsi l'épiphanie est tout entière dans le chant et dans le rite que le chant imite jusqu'à l'illusion. Elle n'est jamais clairement un référent, un objet que le discours pourrait désigner ou éclairer. Même les jeunes filles qui sont invitées à s'activer autour de l'idole et désignées pour recevoir le choc de l'épiphanie divine ne paraissent pas en recueillir la réalité ni le bénéfice. Elles ne sont que les représentantes d'une instance qui les dépasse, Argos, terre et peuple, ici incarnés par la voix énonciatrice. Toute une mise en scène vise à cette rencontre entre une divinité et un peuple, l'une et l'autre nécessairement représentés par leur signe. C'est là précisément que réside l'émotion religieuse. Mais pour qui prétend voir et toucher, réception rime, ici encore, avec déception.

Or ce procédé d'inclusion de la figure mythique du dieu dans l'action rituelle représentée au moment précis d'une manifestation divine paraît exister dans l'image, sous des formes qui n'offrent pas d'épiphanie a priori mais qui n'en sont pas moins des expressions très fortes de la présence du destinataire du culte, en tant que cette présence est à la fois le produit nécessaire du rite et la libre venue du dieu. Nous en citerons deux exemples.

Un cratère à volutes apulien à figures rouges du Peintre de la Naissance de Dionysos (410/380 av. J.-C. $)^{26}$ montre une scène de sacrifice à Dionysos au moment précis où une femme porteuse d'une nébride et ceinte d'une couronne, derrière un autel décoré d'un bucrane à stemmata, où sa position la place aux côtés mêmes de la statue archaïque du dieu porte-thyrse, va égorger un chevreau qu'elle tient, couteau pointé vers la gorge, juste au-

${ }^{26}$ Naples, MN 2411 (inv. 82922), cf. A. D. Trendall et A. Cambitoglou, The redfigured vases of Apulia, I, 1978-1982, p. 35, 2/8; LIMC III Dionysos 863; et F. T. van Straten, Hiera kala. Images of animal sacrifice in archaic and classical Greece, Leyde, 1995, fig. 111 [V149] et p. 105. 
dessus du foyer allumé. Une œnochoé est placée sur une trapeza à droite de l'autel sur l'image; plus à droite encore, une autre femme s'approche, portant un plateau contenant des gâteaux et d'autres offrandes représentés avec précision. Sur la gauche, deux femmes se livrent à un danse agitée, l'une portant des crotales, l'autre un tambourin. Au-dessus de la scène, Dionysos «lui-même» est peint nu, ceint d'une couronne, mollement assis sur son vêtement et l'épaule appuyée sur une longue branche portant encore, à son extrémité, feuillages et bandelettes, où il repose sa main droite; un satyre et quatre ménades l'environnent, en des postures et des activités variées. L'extraordinaire de cette scène est d'abord qu'elle est l'une des très rares à illustrer le moment de l'égorgement sacrificiel, ensuite que le sacrificateur est ici une femme. Nous sommes loin des gestes réglés du sacrifice ordinaire : ce geste de mise à mort et ces danses, c'est Dionysos qui les (dé) règle. Il est présent, quoique invisible, dans les femmes qu'il possède. Aussi cette présence, réelle dans le geste rituel lui-même, peut-elle être ici indiquée doublement, sous la forme de la statue frontale d'une part, du dieu «de chair» spectateur, ensuite.

Un stamnos attique à figures rouges attribué au peintre Polygnote $(450 / 425 \text { av. J.-C. })^{27}$ nous présente une de ces scènes synoptiques où l'on voit se concentrer autour de l'autel toute une série de gestes et d'événement rituels dans ce qui semble être le sommet du sacrifice sanglant tel que l'illustre l'iconographie des vases : le moment où, tandis que des acolytes font griller les viscères au bout de broches tenues au-dessus du feu, l'osphus, déposé dans les flammes, et qu'on prend bien garde de ne pas toucher avec les broches (Ar., Paix 1053), se recourbe en son extrémité et vient pointer vers le ciel, cependant que le sacrifiant fait un geste de prière (main gauche levée) ou d'accueil (main tendue vers l'osphus) tout en esquissant une libation $^{28}$. Ici, Diomède sacrifiant tient une phiale où, porteuse d'une œnochoé, une Victoire voltigeant au-dessus de l'autel verse le vin. Or, Apollon (les noms sont inscrits sur l'image) se tient debout à droite, sur le même plan et de même taille que le sacrifiant, couronné de laurier, une cithare à la main gauche. Comment comprendre cette figuration de la présence d'Apollon, qui d'ailleurs ne suscite pas de réaction des acteurs humains? L' épiphanie n'est pas là où on croit : elle est dans le recourbement attendu de l'osphus, dont Prométhée enchaîné explique, dans Eschyle (v. 496-499), qu'il a appris aux hommes à lire la signification divinatoire, et qui traduit l'acceptation du sacrifice par la divinité destinataire. Aussi certaines figurations

${ }^{27}$ CVA : Gotha, 2, (Deutschland 29) 1968, pl. 56-58; LIMC II Apollon 953; LIMC III Diomedes I 3; F. van Straten, Hiera kala, fig. 132 [V179] et p. 135.

${ }^{28}$ Cf. dans F. van Straten, op. cit., fig. 130-140. 
d'une telle scène montrent-elles les acteurs dardant vers l'osphus un œil avide et fasciné : le miracle (nécessaire d'ailleurs, et néanmoins événement merveilleux) vient de se produire, on peut déclarer que hiera kala, «le sacri-

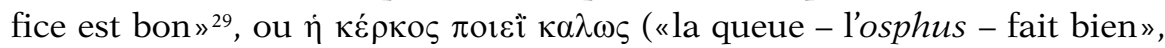
Ar., Paix 1054-55). Apollon est-il ici le destinataire qui se prononce ainsi sur l'agrément du sacrifice? Ou bien est-il l'instance divinatoire présidant à ce moment-là du sacrifice, comme pourraient également le suggérer des figurations d'un laurier derrière l'autel dans des images parallèles ${ }^{30}$ ? La Victoire qui plane est-elle l'indice d'une victoire obtenue par le sacrifiant ou par le destinataire du vase? Il est difficile de trancher. L'essentiel est pour nous que, dans un moment de grande intensité rituelle et émotionnelle (comme le prouvent les regards et les gestes), le dieu soit montré présent à et dans l'action et l'événement cultuels.

Renée Piettre

${ }^{29}$ Ibid., p. 190 sq.

${ }^{30}$ Ibid., fig. 138 et 139 . 